MATHEMATICS OF COMPUTATION

Volume 69, Number 232, Pages 1435-1455

S 0025-5718(00)01238-2

Article electronically published on March 6, 2000

\title{
A HIERARCHICAL METHOD FOR OBTAINING EIGENVALUE ENCLOSURES
}

\author{
E. B. DAVIES
}

\begin{abstract}
We introduce a new method of obtaining guaranteed enclosures of the eigenvalues of a variety of self-adjoint differential and difference operators with discrete spectrum. The method is based upon subdividing the region into a number of simpler regions for which eigenvalue enclosures are already available.
\end{abstract}

\section{INTRODUCTION}

A rigorous method of obtaining enclosures of the eigenvalues of self-adjoint operators has recently been described by Goerisch and Plum [5, 8, 9, 10]. It depends upon having a soluble comparison operator, from which a controlled homotopy is carried out. In this paper we introduce a new method which has the advantage of not requiring such a comparison operator, and apply it to a variety of examples. In Sections 2-5 we consider Sturm-Liouville operators in some detail. Sections 6 and 7 describe how to adapt the method to higher order operators and systems, still in one dimension, and in Sections 8 and 9 we treat discrete Laplacians on graphs. Section 10 describes briefly how to modify the method in order to obtain spectral enclosures for the Laplacian acting in a bounded region in Euclidean space and other elliptic differential operators with variable coefficients; further details and the associated code have been developed elsewhere [1.

We distinguish between computing an eigenvalue in floating point arithmetic, and obtaining guaranteed enclosures. When using the word "enclosure" we shall always understand that the calculation is mathematically rigorous, and that the computations are done in interval arithmetic. Most numerical computations do not give proofs that the values obtained are correct, but depend upon the experience of the person who writes or uses the program concerning its range of reliability. With guaranteed enclosures on the other hand, the value obtained is known to be correct within the stated error bounds, unless there is an actual error at some stage of the computation.

There are already several methods of computing the eigenvalues of a SturmLiouville operator $H$ acting in $L^{2}(\alpha, \beta)$, and higher order analogues. The most obvious one, called shooting, solves the initial value problem for the eigenvalue equation $H f=\lambda f$ subject to the given boundary conditions at $\alpha$ and then varies $\lambda$

Received by the editor October 27, 1998.

1991 Mathematics Subject Classification. Primary 34L15, 35P15, 49R05, 49R10, 65L15, 65L60, $65 \mathrm{~L} 70,65 \mathrm{~N} 25$.

Key words and phrases. Spectrum, eigenvalues, spectral enclosures, interval arithmetic, Rayleigh-Ritz method, Temple-Lehmann method. 
until the boundary condition at $\beta$ is also valid. Most shooting programs do not try to give guaranteed error bounds. Although this is entirely possible [7], the method is difficult to implement numerically if the potential is singular at both ends of the interval.

A second method, introduced by Goerisch [5] and Plum [8, 9, 10, obtains guaranteed enclosures on the eigenvalues of a self-adjoint operator $H$ by a continuous homotopy method, starting from a simpler operator. This is often exactly soluble, but a minimum requirement is that one can obtain sufficiently good rigorous lower bounds on its eigenvalues. Our method is similar to theirs in that it also uses a homotopy from a simpler operator. However they consider a continuous homotopy in some parameter, which often changes the coefficients smoothly to those of an operator with constant coefficients, while we consider a discrete homotopy in certain internal boundary conditions which we choose to insert. We have compared our variation of the homotopy method with theirs for some of the examples Plum solves, and it appears to be substantially more efficient. In higher dimensions we are able to treat examples which are beyond the earlier method, because of the nonexistence of an exactly soluble operator possessing a continuous homotopy to the given operator.

One may obtain rigorous upper bounds on any specified number of eigenvalues by means of the Rayleigh-Ritz (RR) or variational method 2]. The starting point is the determination of accurate approximations to the eigenfunctions by a nonrigorous auxiliary calculation, possibly an inverse iteration method. Once these have been found, one starts again using RR to obtain rigorous upper bounds on the eigenvalues of the operator $H$ in interval arithmetic.

The lower bound is obtained by the method of Temple-Lehmann (TL) which also depends upon the choice of suitable test functions [2, 3, 10, 11. However, in this case one also needs to have crude lower bounds on the eigenvalues, and these are precisely what is missing at the rigorous level. More precisely if the eigenvalues of $H$, written in increasing order and repeated according to multiplicity, are $\left\{\lambda_{n}\right\}_{n=0}^{\infty}$, then in order to obtain an accurate lower bound on $\lambda_{n}$ for some $n$ using TL one needs already to be in possession of a number $\rho$ such that

$$
\lambda_{n}<\rho<\lambda_{n+1},
$$

where $\rho$ is not too close to $\lambda_{n}$. There are three possible methods of obtaining such lower bounds.

(i) One might hope that the upper bound on $\lambda_{n+1}$ is fairly accurate and take $\rho$ to be a slightly smaller number. This idea cannot be turned into a rigorous procedure and will not be discussed further.

(ii) One can use the Goerisch-Plum coefficient homotopy method of obtaining enclosures for many operators.

(iii) One can use a boundary condition homotopy method. The description of this new method is the main contribution of this paper.

In the above we have not mentioned the extra complications which arise if $\lambda_{n}$ is degenerate or nearly so. There are well-known modifications of TL which deal with this problem [2, 8, 11], but we did not want to over-complicate the discussion at this stage. 


\section{NeUmann DECOUPLing}

Let $H$ be a Sturm-Liouville operator acting in $L^{2}(\alpha, \beta)$. We assume that $H$ is of the form

$$
H f(x):=-\frac{\mathrm{d}}{\mathrm{d} x}\left\{a(x) \frac{\mathrm{d} f}{\mathrm{~d} x}\right\}+V(x) f(x),
$$

where $a$ is a positive function in $C^{1}[\alpha, \beta]$ and $V \in L^{\infty}[\alpha, \beta]$. We assume Neumann boundary conditions (NBC) in order to emphasize that the method does not depend upon the very strong monotonicity properties which hold for Dirichlet boundary conditions [2].

Our method is based upon decoupling the interval $(\alpha, \beta)$ into $2^{N}$ subintervals; in most examples considered by the author one can take $N=3$ or $N=4$. The subintervals do not need to be of equal length, but this is the easiest choice to make. We put

$$
\alpha=\alpha_{0}<\alpha_{1}<\cdots<\alpha_{2^{N}}=\beta
$$

and let $H_{i}$ denote the restriction of $H$ to $L^{2}\left(\alpha_{i-1}, \alpha_{i}\right)$ subject to NBC. We then define $A_{N}$ to be the sum of the $H_{i}$, so that $A_{N}$ once again acts in $L^{2}(\alpha, \beta)$. The operators $H$ and $A_{N}$ have the same quadratic form

$$
Q(f):=\int_{\alpha}^{\beta}\left\{a(x)\left|f^{\prime}(x)\right|^{2}+V(x)|f(x)|^{2}\right\} \mathrm{d} x
$$

but with different quadratic form domains $\mathcal{Q}(H)$ and $\mathcal{Q}\left(A_{N}\right)$. The space of all $C^{1}$ functions on $[\alpha, \beta]$ is a quadratic form core for $H$, but to obtain a quadratic form core for $A_{N}$ one must allow the functions to have arbitrary jump discontinuities at each $\alpha_{i}$. Since $\mathcal{Q}(H) \subset \mathcal{Q}\left(A_{N}\right)$, the RR method [2] shows that the eigenvalues of $A_{N}$ are less than or equal to those of $H$.

We define intermediate operators $A_{n}$ acting in $L^{2}(\alpha, \beta)$ for $0 \leq n \leq N$, by a similar method. The component operators of $A_{n}$ are similar to those of $A_{N}$ but only using the points $\alpha_{i}$ where $i=j \cdot 2^{N-n}$ for $0 \leq j \leq 2^{n}$. At each stage the quadratic form domain decreases, leading to the operator inequalities

$$
A_{N} \leq A_{N-1} \leq \cdots \leq A_{0}=H
$$

The following theorem enables the eigenvalues of $A_{n}$ to be computed rigorously using TL once one knows those of $A_{n+1}$. Since the passage from $A_{n+1}$ to $A_{n}$ consists of putting together intervals in pairs, and the set of eigenvalues of $A_{n}$ is simply the collection of all eigenvalues of its component operators $H_{i}$, it is sufficient to deal with the following special case, which also describes the passage from $A_{1}$ to $A_{0}=H$.

Let $\alpha<\gamma<\beta$ and let $\left\{\lambda_{i}\right\},\left\{\mu_{i}\right\},\left\{\nu_{i}\right\}$, denote the eigenvalues of the operators $H_{1}, H_{2}$ and $H$ associated with the intervals $(\alpha, \gamma),(\gamma, \beta),(\alpha, \beta)$, respectively, all subject to NBC. Finally let

$$
\left\{\sigma_{i}\right\}:=\left\{\lambda_{i}\right\} \cup\left\{\mu_{i}\right\}
$$

subject to re-ordering in increasing order and repeating according to multiplicities. Then $\left\{\sigma_{i}\right\}$ are the eigenvalues of the operator $A:=H_{1}+H_{2}$.

Theorem 1. The eigenvalues $\left\{\sigma_{i}\right\}$ and $\left\{\nu_{i}\right\}$ interlace in the sense that

$$
\sigma_{i} \leq \nu_{i} \leq \sigma_{i+1}
$$


for all $i$. Moreover, these are strict inequalities unless the derivative of the relevant eigenfunction of $H$ vanishes at the point $\gamma$.

Proof. The idea is that $H$ differs from $A$ by a rank one perturbation in a certain singular sense. More precisely let $s<\sigma_{0}$ and compare $(A+s)^{-1}$ with $(H+s)^{-1}$. Both have Green functions which can be computed from the two fundamental solutions of the differential equation

$$
-\frac{\mathrm{d}}{\mathrm{d} x}\left\{a(x) \frac{\mathrm{d} f}{\mathrm{~d} x}\right\}+V(x) f(x)+s f(x)=0 .
$$

If one computes the difference of the two kernels one finds that it is a rank one operator. The inequality which we want is equivalent to

$$
\left(\sigma_{i}+s\right)^{-1} \geq\left(\nu_{i}+s\right)^{-1} \geq\left(\sigma_{i+1}+s\right)^{-1},
$$

and this holds whenever one has a positive rank one perturbation by an application of the min-max principle. For an alternative proof see Theorem 5.

The eigenvalues of $H_{1}$ are all distinct, as are the eigenvalues of $H_{2}$, because $H_{i}$ are Sturm-Liouville operators. However there may be coincidences between the two sets of eigenvalues, which imply that $\sigma_{i}=\sigma_{i+1}$. This is not a serious problem, but it can usually be avoided if desired by moving the point $\gamma$ slightly. Assuming that this has been done it is not possible that $\sigma_{i}=\nu_{i}$ : this equality would imply that the corresponding eigenfunction of $H$ happens to have zero derivative at $\gamma$, in which case it is also an eigenfunction for both $H_{1}$ and $H_{2}$, so $\sigma_{i}=\sigma_{i+1}$.

\section{The ENCLOSURE ALGORITHM}

We start with a subdivision of $(\alpha, \beta)$ into $2^{N}$ parts which is fine enough for us to be able to obtain disjoint enclosures on the eigenvalues of each component $H_{i}$ by comparison with constant coefficient operators. From here onward we suppose that we are only interested in obtaining enclosures on those eigenvalues of $H$ which are less than some preassigned number $E$. The larger the value of $E$, the larger one must take $N$ in order to be able to start the procedure. The following lemma shows that it is sufficient for the coefficients to be close to constant in each interval. We only consider the case of $H$ itself for notational simplicity, but the lemma should actually be applied to each component $H_{i}$ of $A_{N}$.

Lemma 2. Suppose that $a_{0} \leq a(x) \leq a_{1}$ and $v_{0} \leq V(x) \leq v_{1}$ for all $x \in(\alpha, \beta)$. Then the eigenvalues $\left\{\lambda_{i}\right\}$ of $H$ satisfy

$$
a_{0} \pi^{2} i^{2} /(\beta-\alpha)^{2}+v_{0} \leq \lambda_{i} \leq a_{1} \pi^{2} i^{2} /(\beta-\alpha)^{2}+v_{1} .
$$

These enclosure intervals are disjoint for all eigenvalues less than a given number $E^{\prime}$ if

$$
0 \leq v_{1}-v_{0} \leq a_{0} \pi^{2} /(\beta-\alpha)^{2}
$$

and

$$
0 \leq v_{1}-v_{0} \leq M^{2} a_{0} \pi^{2} /(\beta-\alpha)^{2}-(M-1)^{2} a_{1} \pi^{2} /(\beta-\alpha)^{2},
$$

where $M$ is the smallest integer such that

$$
E^{\prime} \leq v_{0}+M^{2} a_{0} \pi^{2} /(\beta-\alpha)^{2} .
$$


Proof. The first inequality follows by comparing $H$ with the obvious constant coefficient operators, whose eigenvalues are exactly computable. The proof of the second uses the observation that

$$
v_{0}+m^{2} a_{0} \pi^{2} /(\beta-\alpha)^{2}-v_{1}-(m-1)^{2} a_{1} \pi^{2} /(\beta-\alpha)^{2}
$$

is a concave function of $m$ which must therefore take its minimum value on the interval $[1, M]$ at one of its ends.

Note. Since the initial intervals $\left(\alpha_{i-1}, \alpha_{i}\right)$ are quite short, the integer $M$ in Lemma 2 may be quite small and Lemma 2 may not impose strong conditions on the constants $v_{0}, v_{1}, a_{0}, a_{1}$.

The algorithm for obtaining enclosures of the eigenvalues of $H$ has several stages.

Stage 1. We have to choose an initial subdivision of the interval $(\alpha, \beta)$ such that each of the subintervals $\left(\alpha_{i-1}, \alpha_{i}\right)$ satisfies the conditions of Lemma 2 . This can be done in several ways, and is discussed further in Section 4.

Stage 2. We choose a number $E^{\prime}>E$, for example $E^{\prime}:=9 E / 8$, and put $E_{n}:=$ $E+n\left(E^{\prime}-E\right) / N$ for all $0 \leq n \leq N+1$.

Stage 3. We subdivide $(\alpha, \beta)$ as described above for a value of $N$ which is large enough for us to obtain disjoint intervals which enclose each of the eigenvalues of each component $H_{i}$ of $A_{N}$ up the number $E_{N+1}$.

Stage 4. We use RR and TL to obtain accurate enclosures of each of the eigenvalues of each component $H_{i}$ up to the number $E_{N}$. Putting these together in pairs we obtain rough enclosures of the eigenvalues of each component $H_{j}$ of $A_{N-1}$ by virtue of Theorem 1 . These enclosure intervals overlap very slightly because the previous accurate enclosures were not perfect.

Stage 5. We apply RR to each component $H_{j}$ of $A_{N-1}$ to obtain smaller upper bounds on each of the eigenvalues of each $H_{j}$ and so to convert the above into rough but nevertheless disjoint enclosures of the eigenvalues of each $H_{j}$ up to $E_{N}$.

Stage 6. We apply TL to obtain accurate enclosures of each of the eigenvalues of each component $H_{j}$ of $A_{N-1}$ up to $E_{N-1}$.

Stage 7 . We repeat the process inductively until we reach accurate enclosures of each of the eigenvalues of $H$ up to $E$.

Some comments are in order.

The introduction of the sequence $E_{n}$ at Stage 1 is needed because TL requires a significant gap above any eigenvalue to be estimated. If there is an eigenvalue very close to the upper limit $E_{n}$ at any stage then that eigenvalue cannot be estimated accurately.

When the eigenvalues of two adjacent operators are combined in Stage 3 it may happen that two eigenvalues of the new list created coincide to a high degree of accuracy. This is one possible cause of the problem mentioned in the next paragraph.

The procedure in Stage 5 may occasionally fail because RR may not decrease the upper bound on an eigenvalue enough to make the intervals disjoint. This is handled by using a higher order version of TL whenever this occurs. In principle this could occur for all eigenvalues, in which case the algorithm might halt, but this is extremely unlikely unless there is a symmetry of the underlying problem, which should have been taken into account before starting the computation. 
Ultimately we do not guarantee either that the algorithm finishes or that the results which it yields are of the desired accuracy, but only that if the algorithm does finish, then the enclosures obtained are correct. If the enclosures are not sufficiently accurate, then one must start again with a larger test function space.

Although we specified that the second order coefficients $a(x)$ of the differential operator should be $C^{1}$, there is no difficulty in accommodating simple jump discontinuities. Once one has determined the location of these points, they should be included in the partition $\left\{\alpha_{i}\right\}_{i=0}^{2^{N}}$ of the interval $(\alpha, \beta)$. The discontinuity of $a(x)$ at a point $\gamma$ imposes an effective internal boundary condition on $H$ at $\gamma$, which must be taken into account when specifying its operator domain, but has no effect on its quadratic form domain.

One way of estimating the total computational effort is to count the number of distinct operators for which we have to compute some of the eigenvalues accurately. At the level $n$ this is $2^{n}$, so the total number is $2^{N+1}-1$.

Since parallel machines will become more important, it should be noted that the computations of the eigenvalues of the different operators $H_{j}$ at any particular level are entirely independent, and may be carried out simultaneously. Thus on a parallel machine the total computational effort is proportional to $N+1$. In all the examples we have considered this means the algorithm has only three or four steps!

Both of the above estimates of computational effort are too pessimistic. At the higher levels it may be seen in the examples we analyse below that the number of eigenvalues of each operator to be computed is very small, because the eigenvalues are far apart. So the computation is much faster at the higher levels than indicated above, whether or not one has a parallel machine.

It is clear that the same procedure may be used irrespective of the boundary conditions at $\alpha$ and $\beta$. It may also be applied to potentials which are singular at the end points provided one has crude bounds on the eigenvalues to replace those of Lemma 2. Its extension to systems and to higher order differential operators is described in Sections 6 and 7.

\section{The subdivision of $(\alpha, \beta)$}

We have suggested above that the subdivision of $(\alpha, \beta)$ should be defined by

$$
\alpha_{i}:=\alpha+i(\beta-\alpha) / 2^{N}
$$

for $0 \leq i \leq 2^{N}$, where the size of $N$ is determined by Lemma 2 as indicated in the algorithm. However it is possible that when combining two eigenvalue lists one finds that two eigenvalues coincide or are undesirably close. This is not an insuperable problem since one can use a higher order version of TL to obtain the required lower bounds on the eigenvalues. However, there is a systematic way of avoiding it, unless one of the eigenfunctions has an interval of constancy.

We first emphasise that there is no hope of obtaining accurate enclosures of the eigenvalues of $H$ unless there is some other nonrigorous method of computing the eigenvalues, such as unsupplemented RR or shooting, which in fact give good approximations to the eigenvalues and eigenfunctions. We use these computed eigenfunctions to choose the bisection point $\gamma$ of $(\alpha, \beta)$ as described below. We then do the same for both of the subintervals $(\alpha, \gamma)$ and $(\gamma, \beta)$ and so on until we have produced a fine enough subdivision of $(\alpha, \beta)$ according to the criterion of Lemma 2. If we have misled ourselves about the best choice of the points $\alpha_{i}$, then nothing is lost because we can still use the above algorithm. If, however, the 
approximations to the eigenfunctions are accurate enough, then the method we now describe will have prevented the problem mentioned above.

Let us suppose that there are $k+1$ eigenvalues of $H$ less than $E$, and that the corresponding eigenfunctions $f_{r}$ have zero derivatives at $p(r)$ points for each $0 \leq r \leq k$. Then we choose $\gamma$ somewhere near the center of $(\alpha, \beta)$ but not at or near to any of the above points. Since there are $P:=p(0)+\cdots+p(k)$ such points altogether, there exists $\gamma \in(3 \alpha / 4+\beta / 4, \alpha / 4+3 \beta / 4)$ which is at a distance at least $(\beta-\alpha) / 4 P$ from each of the points.

Whether or not it is worth using this iterative procedure for selecting the subdivision of $(\alpha, \beta)$ remains to be seen. In the two cases solved below, it appears that using a uniform subdivision is perfectly satisfactory.

There is an entirely different reason for choosing a nonuniform subdivision of $(\alpha, \beta)$, if the coefficients of $H$ vary substantially from one part of the interval to another. If the potential is bigger than the number $E$ in some interval, then one should make that entire interval one of the $\left(\alpha_{i-1}, \alpha_{i}\right)$, however big it is, because there will be no relevant eigenvalues associated with it. More generally the size of each interval $\left(\alpha_{i-1}, \alpha_{i}\right)$ should be as big as possible subject to being able to obtain disjoint enclosures of all of the eigenvalues of $H_{i}$ less than $E$. This procedure reduces the number of operators for which one has to compute some of the eigenvalues. A more thorough investigation might involve the uncertainty principle, but Lemma 2 suffices for most purposes.

\section{EXAMPLES}

We illustrate our general theory with two numerical examples, which are solved using shooting and floating point arithmetic, not using interval arithmetic as is actually required. There are two reasons for this, the first being that our goal here is only to examine the feasibility of the method, not to create a new software package. The second is that one should not use a high-powered technique for obtaining eigenvalue enclosures until one has a good idea of the approximate location of the eigenvalues. This information cannot be used in the final computation because it is not rigorous, but it may indicate problems which need special attention in the rigorous computation.

The two examples were studied in detail by Plum [8] using a continuous homotopy in the coefficients.

Example 3. Let $H$ be the operator defined by

$$
H f(x):=-\frac{\mathrm{d}^{2} f}{\mathrm{~d} x^{2}}+8 \cos (x)^{2} f(x)
$$

acting in $L^{2}(0, \pi)$ subject to NBC. Plum obtained enclosures on the eigenvalues ranging from

$$
\mu_{0}=2.48604311_{47}^{50}
$$

to

$$
\mu_{8}=68.03175_{6}^{8}
$$

using his homotopy method, RR and TL, and interval arithmetic. Putting $N=$ 2 the conditions of, Lemma 2 are satisfied for any choice of $E$ for each of the components $H_{i}, 1 \leq i \leq 4$, with $v_{1}-v_{0}=4, a_{0}=a_{1}=1$ and $\alpha_{i}-\alpha_{i-1}=\pi / 4$. Now let $K_{1}, K_{2}$ be the two operators at level one, acting in the intervals $(0, \pi / 2)$ 
and $(\pi / 2, \pi)$. We have computed the eigenvalues of all of the operators above up to the limit $E=70$.

For $i=1,4$, Lemma 2 yields the crude enclosures

$$
4<\mu_{0}<8, \quad 20<\mu_{1}<24, \quad 68<\mu_{2}<72,
$$

while more accurate, but nonrigorous, calculations provide

$$
\mu_{0} \simeq 6.454, \quad \mu_{1} \simeq 22.450, \quad \mu_{2} \simeq 70.515 .
$$

For $i=2,3$, Lemma 2 yields the crude enclosures

$$
0<\mu_{0}<4, \quad 16<\mu_{1}<20, \quad 64<\mu_{2}<68, \quad 144<\mu_{3},
$$

while more accurate calculations yield

$$
\mu_{0} \simeq 1.364, \quad \mu_{1} \simeq 17.693, \quad \mu_{2} \simeq 65.503 .
$$

We now join together the eigenvalue lists of $H_{1}$ and $H_{2}$ to obtain the list

$$
1.364, \quad 6.454, \quad 17.693, \quad 22.450, \quad 65.503, \quad 70.515 \text {. }
$$

If these values are indeed accurate, then according to Theorem 1 they interlace the eigenvalues of $K_{1}$ and provide the basis for the use of RR and TL for obtaining accurate enclosures of the eigenvalues of $K_{1}$. The eigenvalues of $K_{1}$ are (again nonrigorously)

$$
2.486, \quad 9.173, \quad 20.141, \quad 40.057, \quad 68.032 \text {. }
$$

These coincide with the eigenvalues of $K_{2}$ since we have not made use of the symmetry of the operator about $x=\pi / 2$. When we combine this list with a second copy of itself, the resulting list interlaces the eigenvalues of $H$, namely

$2.486, \quad 6.397, \quad 9.173, \quad 13.370, \quad 20.141, \quad 29.084, \quad 40.057, \quad 53.042, \quad 68.032$.

It would have been possible to avoid the coincidence of the eigenvalues of $K_{1}$ and $K_{2}$ by starting with the partition $0,0.6,1.2,2.1, \pi$ instead of the partition into equal subintervals.

The above computation involves determining the eigenvalues of 7 operators at 3 different levels. At the top level we only had to compute the first 3 eigenvalues of each operator $H_{i}$, at the middle level we had to compute 5 and at the bottom level we had to compute 9 .

By comparison Plum computed the eigenvalues of 10 intermediate operators, with less scope for parallelization since each computation depended on the previous one. We computed a total of 31 eigenvalues, while Plum computed at least 90. Plum was not, however, particularly concerned with minimizing numerical effort in his paper.

Example 4. We consider the operator

$$
H f(x):=-\frac{\mathrm{d}^{2} f}{\mathrm{~d} x^{2}}+1000 x f(x)
$$

acting on $L^{2}(0,1)$ subject to DBC. This is essentially the same as Example 2 of Plum [8], who obtained enclosures on the eigenvalues ranging from

$$
\mu_{0}=233.8107_{35}^{42}
$$

to

$$
\mu_{9}=1508.10_{78}^{83}
$$


The unpublished enclosures of Lohner [7], obtained by shooting, are considerably more accurate. We put $N:=3, \alpha_{i}:=i / 8$ for $0 \leq i \leq 8$ and $E:=1000$. A modification of Lemma 2 to cope with the DBC at 0,1 yields the following crude eigenvalue enclosures.

In $(0,1 / 8)$ we have initially

$$
157<\mu_{0}<283, \quad 1421<\mu_{1}<1547
$$

and then more accurately

$$
\mu_{0} \simeq 245.225, \quad \mu_{1} \simeq 1486.798 .
$$

In $(1 / 8,1 / 4)$ we have initially

$$
125<\mu_{0}<250, \quad 756<\mu_{1}<882,
$$

and then more accurately

$$
\mu_{0} \simeq 185.471, \quad \mu_{1} \simeq 820.761 .
$$

We omit the results for the other intervals, each of which involves the computation of only two eigenvalues, all higher eigenvalues being bigger than 1500. We now consider level 2. Putting the previous lists together in pairs and considering the interval $(0,1 / 4)$ we obtain the crude bounds

$$
185.471 \leq \mu_{0} \leq 245.225 \leq \mu_{1} \leq 820.761 \leq \mu_{2} \leq 1486.798 \leq \mu_{3} .
$$

In fact the upper bound on each $\mu_{i}$ is slightly bigger than the lower bound on $\mu_{i+1}$, because the number separating them is not exact, but the use of RR reduces the upper bound on each eigenvalue substantially and so yields disjoint enclosures of the eigenvalues (or actually would do so if the calculations were rigorous). All higher eigenvalues are greater than 1500 . The accurate eigenvalues for the interval $(0,1 / 4)$ are

$$
\mu_{0}=205.942, \quad \mu_{1}=490.938, \quad \mu_{2}=1115.419
$$

We omit the further computations at levels 2 and 1. The full list of eigenvalues of $A_{1}$ is

233.705, 400.348, 532.152, 601.881, 748.110, 825.999, 1007.897, and interlaces the list of eigenvalues of $H$, namely

$$
233.811, \quad 408.795, \quad 552.056, \quad 678.679, \quad 794.738, \quad 906.461,
$$

which agree with the enclosures of Plum [8]. We next comment on the amount of computation needed by our method.

The total number of operators considered by our method is 15 , compared with 50 in Plum's method, since he puts $\delta=0.02$. If one has a parallel machine, then the relevant quantity is the number of levels, namely 4 . For each operator at level 3 we needed to compute 1 or 2 eigenvalues. For each operator at level 2 we computed 3 eigenvalues. For the two operators at level 1 we computed 5 and 3 eigenvalues. Finally we computed all 6 eigenvalues of $H$ in the interval [0,1000], making a total of at most 42 eigenvalues computed. Plum's method involves the computation of at least 300 eigenvalues, but he did not attempt to minimize this number. 


\section{HighER ORDER OPERATORS}

The procedure that we described above can be modified to treat higher order differential operators in one dimension. The difference in the higher order case is that decoupling an interval into two parts by introducing a Neumann boundary condition is not equivalent to a rank one perturbation. However it is still of finite rank, as we will now explain.

Let $H$ be defined formally on $L^{2}(\alpha, \beta)$ by

$$
H f(x):=(-1)^{m} \frac{\mathrm{d}^{m}}{\mathrm{~d} x^{m}}\left\{a(x) \frac{\mathrm{d}^{m} f}{\mathrm{~d} x^{m}}\right\},
$$

where $a \in C^{m}[\alpha, \beta]$ is positive. Our method can also deal with more complicated operators involving lower order terms. We assume Neumann boundary conditions, in the sense that we take the quadratic form of the operator to be

$$
Q(f):=\int_{\alpha}^{\beta} a(x)\left|\frac{\mathrm{d}^{m} f}{\mathrm{~d} x^{m}}\right|^{2} \mathrm{~d} x
$$

with domain the Sobolev space $W^{m, 2}(\alpha, \beta)$. It is known that $Q$ is closed on this domain, and we define $H$ to be the nonnegative self-adjoint operator associated with the form in the standard manner.

Functions in $W^{m, 2}(\alpha, \beta)$ are continuous on $[\alpha, \beta]$ along with all derivatives of order less than $m$. Given $\alpha<\gamma<\beta$ we introduce a Neumann boundary condition at $\gamma$ by replacing $W^{m, 2}(\alpha, \beta)$ by the space $\mathcal{Q}_{m}$ in which we allow the functions and their first $m-1$ derivatives to have simple jump discontinuities at $\gamma$. Let $H_{m}$ be the corresponding operator on $L^{2}(\alpha, \beta)$. We now define a chain of operators $H_{r}$ for $0 \leq r \leq m$ with $H_{0}:=H$. Each of them is associated with the same form $Q$ but on different domains $\mathcal{Q}_{r}$. We define $\mathcal{Q}_{r}$ to be the space of functions in $\mathcal{Q}_{m}$ such that all derivatives of $f$ from the order $r$ to $m-1$ inclusive are continuous at $\gamma$. Thus $\mathcal{Q}_{r} \subset \mathcal{Q}_{r+1}$ for all $r$, each being of codimension one in the next.

Theorem 5. Let $H$ and $K$ be two nonnegative self-adjoint operators on a Hilbert space $\mathcal{H}$ such that their quadratic forms coincide on their common domain. Suppose also that the form domain $\mathcal{Q}(K)$ of $K$ is a subspace of codimension 1 in $\mathcal{Q}(H)$. Finally suppose that $H$ and $K$ both have purely discrete spectrum and that their eigenvalues, written in increasing order and repeated according to multiplicity, are $\left\{\lambda_{n}\right\}_{n=0}^{\infty}$ and $\left\{\mu_{n}\right\}_{n=0}^{\infty}$, respectively. Then the two sets of eigenvalues interlace in the sense that

$$
\lambda_{n} \leq \mu_{n} \leq \lambda_{n+1}
$$

for all $n$.

Proof. This is an immediate consequence of the min-max principle, since every subspace of dimension $n$ of $\mathcal{Q}(H)$ is either already contained in $\mathcal{Q}(K)$ or intersects $\mathcal{Q}(K)$ in a subspace of dimension $n-1$.

The application of this theorem to higher order operators is immediate. In order to remove a Neumann boundary condition at the point $\gamma$ we have to pass through a chain of operators $H_{r}$ with $r$ decreasing from $m$ to 0 . At each stage the eigenvalues interlace, and this is the condition needed to apply the TL technique, as described in Section 3.

We have described the operator $H_{r}$ in terms of its quadratic form domain. This is sufficient for the application of the RR technique. However, the TL method 
depends upon the selection of test functions from the operator domain, so we need to describe this. We first comment that functions in any of the operator domains lie in $C^{2 m-1}[\alpha, \gamma]+C^{2 m-1}[\gamma, \beta]$ because of our smoothness assumption on the coefficient $a(x)$. Also the weak derivative $f^{(2 m)}(x)$ in each subinterval must lie in $L^{2}$. Of course eigenfunctions are more regular and must lie in $C^{2 m}[\alpha, \gamma]+C^{2 m}[\gamma, \beta]$.

We now specify the boundary conditions. The choice of quadratic form domain implies that if $f$ lies in the operator domain then $f^{(r)}(x)=0$ for $x=\alpha, \beta$ and for all $m \leq r \leq 2 m-1$, i.e., Neumann boundary conditions. We need to impose $2 m$ boundary conditions at $\gamma \pm$ to obtain a self-adjoint operator, and these are different for each operator $H_{r}$. Our quadratic form assumption is that $f^{(s)}(\gamma-)=f^{(s)}(\gamma+)$ for all $s$ such that $r \leq s \leq m-1$. This corresponds to the assumption that

$$
\begin{aligned}
f^{(s)}(\gamma+) & =f^{(s)}(\gamma-) & & \text { for all } r \leq s \leq m-1 \\
\left(a f^{(m)}\right)^{(s)}(\gamma+) & =\left(a f^{(m)}\right)^{(s)}(\gamma-) & & \text { for all } 0 \leq s \leq m-r-1 \\
\left(a f^{(m)}\right)^{(s)}(\gamma \pm) & =0 & & \text { for all } m-r \leq s \leq m-1
\end{aligned}
$$

for all $f$ in the operator domain of $H_{r}$, as one may see by carrying out some integrations by parts and requiring the boundary terms to vanish.

The test functions chosen for the TL procedure must satisfy all of the above boundary conditions. One could use a space consisting of different polynomials in each subinterval, with the coefficents restricted to satisfy the boundary conditions at $\alpha, \beta, \gamma$, but many other choices are possible.

\section{Systems of ORDINARY DIFFERENTIAL EQUATIONS}

A self-adjoint system of Sturm-Liouville operators is defined as an Operator $H$ acting in $L^{2}\left((\alpha, \beta), \mathbf{C}^{m}\right)$ according to the formula

$$
H f_{i}(x):=-\sum_{j=1}^{m} \frac{\mathrm{d}}{\mathrm{d} x}\left\{a_{i, j}(x) \frac{\mathrm{d} f_{j}}{\mathrm{~d} x}\right\}+\sum_{j=1}^{M} V_{i, j}(x) f_{j}(x)
$$

We assume that $a_{i, j} \in C^{1}[\alpha, \beta]$ and $V_{i, j} \in L^{\infty}[\alpha, \beta]$ for all $i, j$. We assume that both matrices are real symmetric for all $x \in[\alpha, \beta]$ and that $a_{i, j}$ is uniformly positive definite on $[\alpha, \beta]$. We finally assume that the operator satisfies NBC in the obvious sense for systems.

The computation of the eigenvalues of $H$ proceeds as in the scalar case with one exception. Namely the removal of an internal NBC involves a perturbation of rank $m$ rather than of rank 1 as in the scalar case. We deal with this as we did for higher order Sturm-Liouville operators in the last section. Instead of writing out the details in the general case, we solve a simple example, which exhibits the essential features of the general case.

Example 6. Put $m=2$ and $a_{i, j}(x):=\delta_{i, j}$ for all $i, j$. Let $\alpha<0<\beta$ and let $u, v$ be arbitrary nonnegative numbers. Then define the matrix-valued potential $V$ by

$$
V(x):= \begin{cases}\left(\begin{array}{cc}
u & 0 \\
0 & 0 \\
v & v \\
v & v
\end{array}\right) & \text { if } \alpha<x<0 \\
\text { if } 0<x<\beta .\end{cases}
$$

Let $H_{1}, H_{2}, H$ be the operators associated with the above expression acting in the intervals $(\alpha, 0),(0, \beta),(\alpha, \beta)$, respectively, all subject to NBC. Let $K$ be the "same" 
operator acting in the interval $(\alpha, \beta)$, subject to $\mathrm{NBC}$ at $\alpha, \beta$, and the following boundary conditions at 0 , expressed in terms of the operator domain:

$$
\begin{aligned}
& f_{1}(0+)=f_{1}(0-), \\
& f_{1}^{\prime}(0+)=f_{1}^{\prime}(0-), \\
& f_{2}^{\prime}(0+)=0, \\
& f_{2}^{\prime}(0-)=0 .
\end{aligned}
$$

If $A_{1}$ is the operator $H_{1}+H_{2}$ acting in $L^{2}(\alpha, \beta)$, then the quadratic forms of $A_{1}, K, H$ are all given by the expression

$$
Q(f):=\int_{\alpha}^{\beta}\left\{\left|f_{1}^{\prime}\right|^{2}+\left|f_{2}^{\prime}\right|^{2}+\sum_{i, j=1}^{2} V_{i, j}(x) f_{i}(x) \overline{f_{j}(x)}\right\} \mathrm{d} x .
$$

$A_{1}$ has the largest quadratic form domain, $W^{1,2}\left((\alpha, 0), \mathbf{C}^{2}\right)+W^{1,2}\left((0, \beta), \mathbf{C}^{2}\right)$, while $H$ has the smallest quadratic form domain $W^{1,2}\left((\alpha, \beta), \mathbf{C}^{2}\right)$, of codimension 2 in the previous one. In between these lies the quadratic form domain of $K$, which is the set of $f \in W^{1,2}\left((\alpha, 0), \mathbf{C}^{2}\right)+W^{1,2}\left((0, \beta), \mathbf{C}^{2}\right)$ such that $f_{1}(0+)=f_{1}(0-)$.

Since each quadratic form domain is a subspace of codimension 1 of the previous one, the eigenvalues of the operators interlace in the sense of Theorem 5 . The eigenvalues of $H_{1}, H_{2}$ are exactly computable, so these observations allow us to obtain enclosures of the eigenvalues of $H$ using RR and TL in the standard manner.

We have chosen this example because the eigenvalues of all four operators involved are essentially exactly computable, and it is easy to confirm the interlacing property directly. We put $\alpha=-1, \beta:=2, u=2 v=100$, and compute all of the eigenvalues of each operator up to $E:=50$.

The eigenvalues of $H_{1}$ consist of all numbers of the form $u+n^{2} \pi^{2} / \alpha^{2}$ or $m^{2} \pi^{2} / \alpha^{2}$, where $m, n$ are nonnegative integers. This yields the list

$$
0,9.870, \quad 39.478, \quad 88.826 \text {. }
$$

The eigenvalues of $H_{2}$ consist of all numbers of the form $2 v+n^{2} \pi^{2} / \beta^{2}$ or $m^{2} \pi^{2} / \beta^{2}$, where $m, n$ are nonnegative integers. This yields the list

$$
0, \quad 2.467, \quad 9.870, \quad 22.207, \quad 39.478, \quad 61.685, \quad 88.826 \text {. }
$$

The eigenvalues of $A_{1}$ are obtained by combining these two lists to obtain

0, $0, \quad 2.467, \quad 9.870, \quad 9.870, \quad 22.207, \quad 39.478, \quad 39.478, \quad 61.685, \quad 88.826$.

The eigenfunctions of $K$ and $H$ are linear combinations of trigonometric and exponential functions, and the eigenvalues are obtained by solving certain trancendental equations associated with the boundary conditions at 0 . The eigenvalues of $K$ are approximately

$0, \quad 0.468, \quad 4.298, \quad 9.870, \quad 12.288, \quad 24.757, \quad 39.478, \quad 41.865, \quad 63.639$,

which interlace those of $A_{1}$. The eigenvalues of $H$ are approximately:

$$
0.449, \quad 1.609, \quad 4.735, \quad 11.746, \quad 17.747, \quad 27.360, \quad 41.177,
$$

which interlace those of $K$.

It may be seen that although the eigenvalues do interlace as the theory predicts, the smallest eigenvalue of $H$ is rather close to the second eigenvalue of $K$, a fact which does not help the efficiency of the TL method. The reason for this is that 
the coefficients $u, v$ are rather large, and this has the effect of partially decoupling the two intervals. Accurate lower bounds on the smallest eigenvalue of $H$ can be obtained by using a higher order version of the TL method.

\section{OPERATORS ON GRAPHS}

The method that we have developed for Sturm-Liouville operators may be applied with modifications to elliptic partial differential operators and to discrete Laplacians on graphs. The first application demands the use of the quite complicated machinery associated with the finite element method. We decribe here the second application, which is of independent interest, and also involves the theory of rank 1 perturbations in certain situations.

We define a graph to be a finite set $X$ together with a set $\mathcal{E}$ of directed edges. We assume that if $e:=(x, y) \in \mathcal{E}$, then $\bar{e}:=(y, x) \in \mathcal{E}$. We define the associated Laplacian to be the operator acting on $l^{2}(X)$ with matrix

$$
A_{x, y}:= \begin{cases}-1 & \text { if }(x, y) \in \mathcal{E}, \\ \operatorname{deg}(x) & \text { if } x=y, \\ 0 & \text { otherwise }\end{cases}
$$

where $\operatorname{deg}(x):=\#\{y \in X:(x, y) \in \mathcal{E}\}$ is the degree of $x$.

The quadratic form corresponding to this matrix is

$$
Q(f):=\frac{1}{2} \sum_{(x, y) \in \mathcal{E}}|f(x)-f(y)|^{2},
$$

which is a nonnegative Dirichlet form, with all of the structural consequences of this fact. We follow standard practice in referring to the eigenvalues of the operator $A$ defined above as eigenvalues of the graph $X$.

Although the matrix $A$ is finite the determination of its eigenvalues is not straightforward if the graph is very large, and one actually has the same problems in obtaining guaranteeed enclosures as for infinite-dimensional problems. The first main problem is the nonexistence of standard comparison problems. There are very few finite graphs for which one can compute the eigenvalues exactly, and there is no possibility of using a change of variables to map a graph to a standard soluble one as in the case of partial differential operators.

We present two procedures for obtaining enclosures of eigenvalues of finite graphs. The first applies to the case in which the graph is obtained from one for which one already has enclosures of the eigenvalues by the removal of a small number of chosen vertices. We leave the reader to formulate the corresponding lemma relating to the addition of a small number of vertices.

Lemma 7. Let $Y$ be a subset of $X$ obtained by the removal of a small number of vertices, and let $\mathcal{G}$ be the set of (undirected) edges of $X$ which join points of $Y$ to points of $X \backslash Y$. Then one may compute enclosures of the eigenvalues of $Y$ from those of $X$ in $\#(\mathcal{G})$ homotopy steps.

Proof. Let $\left\{e_{i}\right\}_{i=1}^{n}$ be some enumeration of the edges in $\mathcal{G}$. Let $A_{i}$ be the matrix acting in $l^{2}(X)$ which is obtained from that of $A$ by the removal of the contribution of the edges $e_{1}, \ldots, e_{i}$. Then

$$
A \geq A_{1} \geq \cdots \geq A_{n}
$$


and each matrix is a rank 1 perturbation of the next one in the chain. It follows by an argument similar to that of Theorems 1 and 5 that the eigenvalues of $A_{i}$ interlace those of $A_{i+1}$ for every $i$. This is the fundamental requirement for transferring accurate enclosures of the eigenvalues from $A_{i}$ to $A_{i+1}$ by the RR and TL methods. The operator $A_{n}$ is the direct sum of the discrete Laplacians of $Y$ and $X \backslash Y$. Since we have assumed that $X \backslash Y$ is small, its eigenvalues may be computed independently by a direct procedure. Removing these eigenvalues leaves those of $Y$.

Let $X$ be a finite subset of $\mathbf{Z}^{N}$ for some $N$. The edges of $X$ are defined to be those pairs $x, y \in X$ such that

$$
\sum_{r=1}^{N}\left|x_{r}-y_{r}\right|=1 .
$$

In this situation we have the $\operatorname{bound} \operatorname{deg}(x) \leq 2 N$ for all $x \in X$. The operator $A$ may be considered to be the discrete Laplacian on $X$ subject to Neumann boundary conditions, since 0 is always an eigenvalue of $A$, the corresponding eigenfunction being constant. The multiplicity of the eigenvalue 0 equals the number of connected components of the graph.

Example 8. Let $X \subset \mathbf{Z}^{2}$ be the set

$$
\{(m, n): 1 \leq m \leq k, 1 \leq n \leq k\},
$$

and let $Y$ be obtained by the removal of the set

$$
Z:=\{(1,1),(1,2),(2,1)\} .
$$

Then an enumeration of the (undirected) edges of $\mathcal{G}$ is $e_{1}:=((1,2),(2,2)), e_{2}:=$ $((2,1),(2,2)), e_{3}:=((1,2),(1,3)), e_{4}:=((2,1),(3,1))$. We chose $k:=7$ and computed the smallest 6 eigenvalues of the operators $A_{i}$. The interlacing property is verified. The eigenvalues of $A_{4}$ coincide with those of the discrete Laplacian of $Y$, together with the eigenvalues $0,1,3$ of the Laplacian of $Z$. The numbers in the table below are actually $k^{2}$ times the eigenvalues, so that they may be compared with the eigenvalues of $-\Delta$ on the unit square subject to NBC.

$\begin{array}{ccccccc} & \mu_{0} & \mu_{1} & \mu_{2} & \mu_{3} & \mu_{4} & \mu_{5} \\ A & 0 & 9.705 & 9.705 & 19.410 & 36.898 & 36.898 \\ A_{1} & 0 & 9.515 & 9.705 & 19.142 & 33.499 & 36.898 \\ A_{2} & 0 & 9.361 & 9.574 & 18.367 & 30.187 & 34.782 \\ A_{3} & 0 & 5.868 & 9.540 & 13.119 & 23.836 & 34.571 \\ A_{4} & 0 & 0 & 9.095 & 11.471 & 23.049 & 32.525\end{array}$

The interlacing property states that the number $\lambda$ immediately above any eigenvalue $\mu$ of $A_{i}$ in the table is a lower bound for the next eigenvalue of $A_{i}$. Let us suppose that the values in the first row of the table are close to accurate enclosures of the eigenvalues of $A$, and that all of the other entries in the table are rigorous upper bounds, which we expect to be accurate. Using the interlacing property we deduce that $\mu_{5}\left(A_{1}\right)=36.898$. The fact that the (upper bounds on the) other eigenvalues of $A_{1}$ are widely separated enables us to use TL to confirm that they have been found accurately. Interlacing establishes that $\mu_{5}\left(A_{2}\right) \geq 33.499$, and we confirm that the other eigenvalues of $A_{2}$ have been computed accurately as before. If interval arithmetic has been used, we end up with accurate enclosures of all of the eigenvalues of $A_{4}$ up to and including $\mu_{4}$. The final reason why this procedure 
works is that the entries in the column labelled $\mu_{4}$ and the row labelled $A_{4}$ decrease rapidly enough for TL to be an efficient method.

The above example is purely illustrative: the same procedure can be carried out for values of $k$ which are large enough for the problem of obtaining eigenvalue enclosures to be nontrivial. If at some stage an eigenvalue does not decrease enough from one stage to the next for TL, then either we have to proceed to a lower eigenvalue or we must use a higher order version of TL.

The above method is not suitable for obtaining enclosures of the eigenvalues of a graph which is far from any graph for which eigenvalue enclosures are already known. As a typical example we mention the set of all $(m, n) \in \mathbf{Z}^{2}$ which satisfy all three inequalities $m^{2}+n^{2}<16 d^{2},(m-2 d)^{2}+n^{2}>d^{2}$, and $(m+2 d)^{2}+n^{2}>d^{2}$, where $d$ is a large positive number.

In cases such as the above we combine the continuous homotopy procedure introduced by Goerisch and Plum with the hierarchical homotopy method we introduced for Sturm-Liouville operators. The idea is to subdivide $X$ into several more or less convex parts each of which is small enough that eigenvalue enclosures can be obtained by a direct method. These parts are then joined together in pairs as described below, obtaining eigenvalue enclosures for the larger parts. If the initial subdivision is into $k:=2^{N}$ parts, then after the first stage one has $2^{N-1}$ parts, and the procedure terminates after $N$ stages. It remains to describe how to join together two subgraphs.

Let $X=Y \cup Z$ where $Y, Z$ are disjoint subgraphs, let $\mathcal{G}$ be the set of edges joining points of $Y$ and $Z$, and let $\mathcal{F}$ be the complement of $\mathcal{G}$ in the set $\mathcal{E}$ of all edges of $X$. Given $0 \leq s \leq 1$, let $A_{s}$ be the matrix associated with the quadratic form

$$
Q_{s}(f):=\frac{1}{2} \sum_{(x, y) \in \mathcal{F}}|f(x)-f(y)|^{2}+\frac{s}{2} \sum_{(x, y) \in \mathcal{G}}|f(x)-f(y)|^{2} .
$$

Lemma 9. The eigenvalues of $A_{s}$ are increasing real analytic functions of the parameter $s$. The eigenvalue list of $A_{0}$ is just the union of the two eigenvalue lists of $Y$ and $Z$. At the other end $A_{1}$ is the discrete Laplacian of $X$.

Proof. The first statement is part of received knowledge [6], while the second depends upon the fact that $A_{0}$ is the direct sum of the discrete Laplacians of $Y$ and $Z$.

The procedure for obtaining eigenvalue enclosures for $X$ is similar to that of Goerisch and Plum 5, 8. We consider the operators $A_{s(r)}$ for a large enough chosen sequence $0=s_{0}<s_{1}<\cdots<s_{p}=1$. If we have enclosures of the eigenvalues of $A_{s(i)}$, then these provide lower bounds on the eigenvalues of $A_{s(i+1)}$ which may be adequate to obtain enclosures of the eigenvalues of the latter operator by the $\mathrm{RR}$ and TL procedures. Eigenvalue crossings may occur at certain values of $s$, but these are handled using the higher order TL procedure.

Example 10. Let $X:=Y \cup Z \subset \mathbf{Z}^{2}$ where

$$
\begin{aligned}
Y & :=\{(m, n): 1 \leq x \leq h-1,1 \leq y \leq x\} \\
Z & :=\{(m, n): h \leq x \leq 2 h-1,1 \leq y \leq 2 h-x\}
\end{aligned}
$$

where $h$ is some positive integer. Let the set $\mathcal{E}$ of edges of $X$ be those inherited from $\mathbf{Z}^{2}$ as before. The undirected edges of $\mathcal{G}$ are of the form $(h-1, r),(h, r)$ where 
$1 \leq r \leq h-1$, and we separate the triangle $X$ into two smaller triangles. We list the 7 smallest eigenvalues of $A_{s}$ below for $h:=8$ and $s:=0,0.2,1$. A larger number of values of $s$ were originally computed, but these are the only ones needed.

$\begin{array}{cccccccc} & \mu_{0} & \mu_{1} & \mu_{2} & \mu_{3} & \mu_{4} & \mu_{5} & \mu_{6} \\ A_{0} & 0 & 0 & 0.12061 & 0.15224 & 0.25330 & 0.32139 & 0.46791 \\ A_{0.2} & 0 & 0.04705 & 0.13054 & 0.23249 & 0.27273 & 0.42485 & 0.49950 \\ A_{1} & 0 & 0.07244 & 0.13259 & 0.27719 & 0.33076 & 0.51058 & 0.60389\end{array}$

Each eigenvalue $\mu_{i}$ is a monotonic increasing function of $s$. Suppose that we already know that the numbers in the first row are accurate approximations to the eigenvalues of $A_{0}$, and that the other numbers are rigorous upper bounds to the corresponding eigenvalues, as determined by $\mathrm{RR}$. We use the monotonicity to deduce that $\mu_{6}\left(A_{0.2}\right) \geq 0.46791$. Using TL we then confirm that $\mu_{5}\left(A_{0.2}\right)$ is accurate. Monotonicity implies that $\mu_{5}\left(A_{1}\right) \geq 0.42485$ and we are finally able to confirm the accuracy of $\mu_{j}\left(A_{1}\right)$ for $j=4,3,2,1,0$ in turn by TL. If all of the computations have been done in interval arithmetic, we have obtained enclosures of the eigenvalues of $A_{1}$ from those of $A_{0}$.

The values of $\mu_{1}\left(A_{s}\right)$ for $s=0 \cdots 1(0.1)$ are

$$
\begin{array}{llllll}
0, & 0.03153, & 0.04705, & 0.05559, & 0.06085, & 0.06439, \\
0.06693, & 0.06882, & 0.07030, & 0.07148, & 0.07244 .
\end{array}
$$

This list exhibits a common pattern of rapid increase for small values of $s$ followed by little change for large values. In fact it follows from $\mathrm{RR}$ that $\mu_{1}\left(A_{s}\right)$ is a concave function of $s$, but this need not be true for higher eigenvalues.

A precondition for applying the above method is that eigenvalue enclosures of the parts $X_{1}, \ldots, X_{k}$ into which we subdivide $X$ should already be known. This may be achieved by making each $X_{i}$ small enough so that all of its eigenvalues can be computed by a direct method. If some of the parts are rectangles or one of a very small number of other graphs, then their eigenvalues may be exactly known. The following argument shows that for some purposes we may dispense with knowledge of accurate enclosures of the eigenvalues of the parts entirely. It requires instead an assumption about the geometry of the parts, expressed initially in terms of a lower bound on their first nonzero eigenvalues.

Given $a>0$ we say that a finite graph $(X, \mathcal{E})$ lies in $\mathcal{C}_{a}$ if its first nonzero eigenvalue $\mu_{1}$ satisfies

$$
\mu_{1} \geq \operatorname{ad}(X, \mathcal{E})^{-2},
$$

where $d(X, \mathcal{E})$ is the diameter of the graph. We investigate the geometric significance of this condition in the next section. The value of $b$ in the following theorem indicates how small the individual subsets in a partition of $X$ need to be in order to be able to obtain accurate enclosures of the eigenvalues of $X$ without already possessing accurate enclosures of the eigenvalues of the subsets.

Theorem 11. Suppose that $a>0$ and that $\left\{X_{i}\right\}_{i=1}^{k}$ is a partition of the graph $X$, each subset of which lies in $\mathcal{C}_{a}$. Suppose also that $b>0$ and that

$$
d\left(X_{i}, \mathcal{E}_{i}\right) \leq \frac{1}{b} d(X, \mathcal{E})
$$


for all $1 \leq i \leq k$. Then one may obtain accurate enclosures of all eigenvalues of $X$ less than

$$
E:=a b^{2} d(X, \mathcal{E})^{-2}
$$

by a continuous homotopy method.

Proof. The conditions of the theorem imply that the first nonzero eigenvalue of each part is at least as big as $E$. Let $(Y, \mathcal{F})$ be the union of two of the subgraphs $\left(X_{i}, \mathcal{E}_{i}\right)$, and let $(Y, \overline{\mathcal{F}})$ be obtained by including also those edges of the graph $(X, \mathcal{E})$ which connect the two parts of $Y$. The spectrum of $(X, \mathcal{F})$ below $E$ consists of the eigenvalue 0 with multiplicity 2 and nothing else. This precise if unusual information is enough to obtain accurate enclosures of the spectrum of $(Y, \overline{\mathcal{F}})$ below $E$ by the Goerisch-Plum continuous homotopy method. By a repetition of this method, carried out in a hierarchical manner, one eventually obtains accurate enclosures of the spectrum of $(X, \mathcal{E})$ below $E$.

\section{The Poincaré inequality}

In order to implement the above ideas, one needs to obtain geometric conditions which imply that the first nonzero eigenvalue of a connected graph $(X, \mathcal{E})$ has a lower bound of order $d^{-2}$, where $d$ is the diameter of the graph. Examples in [4] show that this is not always uniformly true for a family of graphs parametrized by the diameter as $d \rightarrow \infty$, but their discrete version of the Poincaré inequality can be rewritten to provide exactly what we need. We develop the theory of this section at a greater level of generality than before, because of its independent interest.

Let $b: \mathcal{E} \rightarrow(0, \infty)$ be a positive weight function satisfying $b(e)=b(\bar{e})$ for all $e \in \mathcal{E}$. Let $|X|$ denote the number of points in $X$. Given a path $\gamma:=\left(\gamma_{1}, \ldots, \gamma_{k}\right)$, we define its length to be

$$
|\gamma|:=\sum_{i=1}^{k} b\left(\gamma_{i-1}, \gamma_{i}\right)^{-1}
$$

and then define the diameter $d$ of $X$ using this notion of length, in the usual manner.

Let $A$ be the operator on $l^{2}(X)$ associated with the quadratic form

$$
Q(f):=\frac{1}{2} \sum_{(x, y) \in \mathcal{E}} b(x, y)|f(x)-f(y)|^{2} .
$$

It is easily seen that 0 is an eigenvalue of $A$ of multiplicity 1 , the corresponding normalized eigenfunction satisfying $\phi_{0}(x)=|X|^{-1 / 2}$ for all $x \in X$.

In order to obtain a lower bound on the first nonzero eigenvalue $\mu_{1}$ of $A$, we follow closely Diaconis and Stroock [4] (and Poincaré). Suppose that $\Gamma$ is a set of paths in $X$, one path from $\Gamma$ joining every ordered pair of points $x, y \in X$. We impose two constraints on the choice of this set of paths. The first, that

$$
\left|\gamma_{x, y}\right| \leq \alpha d
$$

for some $\alpha$ and all $x, y \in X$, is self-explanatory. The second is that

$$
\#\{\gamma \in \Gamma: e \in \gamma\} \leq \beta d|X|
$$

for some $\beta>0$ and all $e \in \mathcal{E}$. Since the total number of paths in $\Gamma$ is $|X|^{2}$, this is a constraint on how well distributed the paths are. The assumption is in precisely the form needed for applications. 
Theorem 12. Under the above two assumptions we have

$$
\mu_{1} \geq \frac{1}{\alpha \beta d^{2}} .
$$

Proof. If $e:=(x, y) \in \mathcal{E}$, we put $\partial f(e):=f(y)-f(x)$. We have

$$
\begin{aligned}
|X|\left\|\phi-\left\langle\phi, \phi_{0}\right\rangle \phi_{0}\right\|^{2} & =\frac{1}{2} \sum_{x, y \in X}|\phi(x)-\phi(y)|^{2} \\
& =\frac{1}{2} \sum_{x, y \in X}\left|\sum_{e \in \gamma_{x, y}} \partial \phi(e)\right|^{2} \\
& \leq \frac{1}{2} \sum_{x, y \in X}\left|\gamma_{x, y}\right| \sum_{e \in \gamma_{x}, y} b(e)|\partial \phi(e)|^{2} \\
& \leq K Q(\phi),
\end{aligned}
$$

where

$$
\begin{aligned}
K: & =\sup _{e \in \mathcal{E}} \sum_{\gamma_{x, y} \ni e}\left|\gamma_{x, y}\right| \\
& \leq \alpha d \#\{\gamma \in \Gamma: e \in \gamma\} \\
& \leq \alpha \beta d^{2}|X| .
\end{aligned}
$$

The proof is completed by using the variational characterisation of $\mu_{1}$

$$
\mu_{1}=\inf \left\{\frac{Q(\phi)}{\left\|\phi-\left\langle\phi, \phi_{0}\right\rangle \phi_{0}\right\|^{2}}: 0 \neq \phi \in l^{2}(X)\right\} .
$$

Upper bounds of a similar type on $\mu_{1}$ are relatively easy to obtain by applying the variational inequality to suitable test functions, but we do not need them here.

The following application of the above theorem is a typical building block for the implementation of the ideas in the last section.

Theorem 13. Define $X \subset \mathbf{Z}^{2}$ by

$$
X:=\{(i, j): 1 \leq i \leq n, 1 \leq j \leq f(i)\},
$$

where $f\{1, \ldots, n\} \rightarrow\{1, \ldots m\}$ is a nondecreasing function. Let $A$ be the discrete Laplacian on $X$, corresponding to the choice $b \equiv 1$ above. Then

$$
\mu_{1} \geq \frac{1}{(n+f(n)-2) \max \{n, f(n)\}} \geq d^{-2},
$$

where $d$ is the diameter of $X$.

Proof. It follows from the definition of $X$ that $d=n+f(n)-2$ and $n \leq|X| \leq n f(n)$. Our main task is to define the set $\Gamma$ of paths. If $i \leq i^{\prime}$, then the path from $(i, j)$ to $\left(i^{\prime}, j^{\prime}\right)$ is the horizontal line from $(i, j)$ to $\left(i^{\prime}, j\right)$, followed by the vertical line from $\left(i^{\prime}, j\right)$ to $\left(i^{\prime}, j^{\prime}\right)$. Because $f$ is monotonic, this is entirely contained in $X$. If $i \geq i^{\prime}$ we take a similar path. It may be seen that every path $\gamma$ has length at most $n+f(n)-2$. The number of paths through any horizontal edge $e \in \mathcal{E}$ is at most $n|X|$, while the number through any vertical edge is at most $f(n)|X|$. A slight modification of the estimate of $K$ in the last theorem completes the proof.

The number of paths through any edge $e \in \mathcal{E}$ can be bounded more efficiently if further information about $f$ is provided. If $f(i):=1$ for $1 \leq i \leq n-1$ and $f(n):=n$, then the theorem yields $\mu_{1} \geq \frac{1}{n(2 n-2)}$ while one actually has $\mu_{1} \sim \frac{\pi^{2}}{4 n^{2}}$ 
for large $n$. It would be valuable to determine the largest constant $c$ such that $\mu_{1} \geq c / n^{2}$ for all graphs of the type described in the above theorem, subject to $f(n) \leq n$. It appears that even the continuous analogue of this problem is unsolved.

\section{The LAPLACIAN IN $N$ Dimensions}

In the applications that we have considered so far, we have taken advantage of the fact that the boundary conditions introduced are associated with finite rank perturbations of the operator. This is not an essential feature of the method. In this section we describe briefly the modifications to the above ideas needed to provide enclosures of the eigenvalues of a partial differential operator. We will consider only the case in which $H:=-\Delta$, acting in $L^{2}(\Omega)$ subject to NBC, where $\Omega$ is a bounded region in $R^{N}$ with piecewise smooth boundary. However, the same method applies to variable coefficient elliptic operators subject to other boundary conditions. By the eigenvalues of any region $\Omega$ we mean the eigenvalues of $-\Delta$ acting in $L^{2}(\Omega)$ subject to NBC.

If the region $\Omega$ is diffeomorphic to the unit ball $B$, Plum 9] has described a method of obtaining enclosures on the eigenvalues by transferring the operator to $L^{2}(B, m(x) \mathrm{d} x)$ where $m$ is a suitable positive weight, and then using his coefficient homotopy method. This cannot be adapted to treat the case in which $\Omega$ contains more than one hole. The method described below is capable of dealing with regions containing any number of holes.

Suppose we wish to find all of the eigenvalues of a region $\Omega$ which are smaller than a given number $E>0$. The first step is to divide the region into subregions $\left\{\Omega_{i}\right\}_{i=1}^{k}$ each with a piecewise smooth boundary. We assume that enclosures of the eigenvalues less than $E$ of each subregion are known, either because its eigenvalues are exactly computable or because it is small enough with a regular enough shape for 0 to be its only eigenvalue below $E$. We also assume that the subregions can be recombined in pairs in a hierarchical fashion to recover the original set $\Omega$. The task therefore is to obtain enclosures of the eigenvalues of the union of two regions which have some common boundary when we already have enclosures of the eigenvalues of the individual regions.

Let $U, V$ be disjoint bounded connected regions in $\mathbf{R}^{N}$ with piecewise smooth boundaries and suppose that their common boundary $B$ is a nonempty $(N-1)$ dimensional surface. Put $\Omega:=U \cup V \cup B$. Suppose also that we have accurate enclosures of all the eigenvalues of each region up to the number $E$. If we combine the two lists of eigenvalues into a single increasing list $\left\{\mu_{i}(0)\right\}$, then this list provides the eigenvalues of the operator $H_{0}=-\Delta$ acting in $L^{2}(\Omega)$ subject to NBC on $\partial U \cup \partial V$. Note that the number 0 is an eigenvalue of multiplicity 2 .

We introduce a family of quadratic forms $Q_{s}$ defined for $0 \leq s<\infty$, all having the same quadratic form domain $\mathcal{D}_{0}:=W^{1,2}(U)+W^{1,2}(V)$. A core for this subspace consists of all functions on $\bar{\Omega}$ which are $C^{1}$ except that they are allowed to be discontinuous as one crosses $B$. Every function $f \in \mathcal{D}_{0}$ has $L^{2}$ boundary values on $B$ which we denote by $f_{ \pm}$depending upon which side of $B$ one approaches it from. We then define

$$
Q_{s}(f):=\int_{\Omega}|\nabla f|^{2}+s \int_{B}\left|f_{+}-f_{-}\right|^{2},
$$

where the second integral is with respect to the natural surface measure on $B$. It is evident that the forms $Q_{s}$ are monotonic increasing. It may be shown that 
the perturbation term is relatively compact, so that the right-hand side is the closed form associated with a certain nonnegative self-adjoint operator $H_{s}$. The eigenvalues of $H_{s}$ are increasing real-analytic functions of $s$ by $[6$.

Since the quadratic forms are monotonic increasing as a function of $s$ they converge to a limit $Q$ defined by

$$
Q(f):=\lim _{s \rightarrow+\infty} Q_{s}(f)
$$

where we adopt the standard convention [2] that $Q(f)=+\infty$ whenever $f$ does not lie in the form domain $\mathcal{D}$ of $Q$. It is clear that

$$
\mathcal{D}=\left\{f \in \mathcal{D}_{0}: f_{+}=f_{-} \text {on } B\right\} .
$$

This space is exactly $W^{1,2}(\Omega)$, so the operator associated with $Q$ is $H:=-\Delta$ acting in $L^{2}(\Omega)$ subject to $\mathrm{NBC}$ on $\partial \Omega$. An immediate consequence is that

$$
\lim _{s \rightarrow+\infty} \mu_{n}(s)=\mu_{n}
$$

for every $n$, the limit being monotone.

Having chosen a suitable increasing sequence $0=s_{0}<s_{1}<\cdots<s_{n}$ one obtains accurate enclosures of the eigenvalues of each $H_{s_{i+1}}$ from those of $H_{s_{i}}$ by the Goerisch-Plum homotopy method. If $s_{n}$ is large enough, then the eigenvalues of $H_{s_{n}}$ will be good enough lower bounds of the eigenvalues of $H$ to enable us to apply RR and TL to obtain accurate enclosures of the eigenvalues of $H$. Many of the details are similar to what we have already discussed, and we concentrate on the novelties.

The upper bounds on all eigenvalues are obtained by RR using a suitable test function space lying in the quadratic form domain $\mathcal{D}_{0}$ or $\mathcal{D}$. An obvious choice is to use a finite element subspace in which the elements are linear, but more sophisticated elements are probably needed for accurate results. The continuity requirement for two elements which have a common edge is suspended if that edge lies within $B$. The restriction of the quadratic form $Q_{s}$ to the test function space can be expanded in terms of the values of the elements at the vertices, noting that there will be two values at each vertex lying on $B$, one corresponding to each side of $B$.

The required lower bounds can only be obtained by TL if one takes a test function space lying in the operator domain, which is different for each operator, even though every operator $H_{s}$ is equal to $-\Delta$ on its own domain. One can use a finite element subspace consisting of $C^{2}$ functions, but this has to respect not only the Neumann boundary condition on $\partial W$ but also certain $s$-dependent internal boundary conditions on $B$.

Let $\partial f_{ \pm}$denote the normal derivatives of $f$ on the two sides of $B$, both taken in the direction from the - side of $B$ to the + side. An application of Gauss' theorem shows that the internal boundary condition is

$$
\partial f_{+}(x)=\partial f_{-}(x)=s\left\{f_{+}(x)-f_{-}(x)\right\}
$$

for all $x \in B$.

It is not possible to demonstrate that the above theory works well in practice, without a substantial amount of effort writing the relevant code. The ideas in this section have been developed further in a paper by Behnke, Plum and Wieners [1], to which we refer for an explicit implementation. 


\section{ACKNOWLEDGMENTS}

We would like to thank M. Plum for several valuable exchanges in the course of this work.

\section{REFERENCES}

[1] H Behnke, M Plum, C Wieners: Eigenvalue inclusions via domain decomposition. Preprint, 1999.

[2] E B Davies. Spectral Theory and Differential Operators. Cambridge Univ. Press, 1995. MR 96h:47056

[3] E B Davies. Spectral enclosures and complex resonances for general self-adjoint operators. LMS J. Comput. Math. 1 (1998) 42-74. CMP 98:15

[4] P Diaconis and D W Stroock: Geometric bounds for eigenvalues of Markov chains. Ann. Appl. Prob. 1 (1991) 36-61. MR 92h:60103

[5] F Goerisch: Ein Stufenverfahren zur Berechnung von Eigenwertschranken. In 'Numerical Treatment of Eigenvalue Problems' vol. 4 ISNM 83 (Ed. J Albrecht et al) pp 104-114. Birkhauser-Verlag, Basel, 1987. MR 90j:65058

[6] T Kato: Perturbation Theory of Linear Operators. Springer-Verlag, Berlin, Heidelberg, New York, 1966. MR 34:3324

[7] R Lohner: Verified solution of eigenvalue problems in ordinary differential equations. Unpublished manuscript, 1990.

[8] M Plum: Eigenvalue inclusions for second order ordinary differential operators by a numerical homotopy method. Z. Angew. Math. Phys. 41 (1990) 205-226. MR 91d:65116

[9] M Plum: Bounds for eigenvalues of second order elliptic differential operators. Z. Angew. Math. Phys. 42 (1991) 848-863. MR 92h:35167

[10] M Plum: Guaranteed numerical bounds for eigenvalues. In 'Spectral Theory and Computational Methods of Sturm-Liouville Problems', eds. D Hinton and P W Schaefer. Marcel Dekker, New York, Basel, 1997. MR 98g:47018

[11] S Zimmermann and U Mertins: Variational bounds to eigenvalues of self-adjoint eigenvalue problems with arbitrary spectrum. Z. Anal. Anwendungen. 14 (1995) 327-345. MR 96d:49046

Department of Mathematics, King's College, Strand, London, WC2R 2LS, United KINGDOM

E-mail address: E.Brian.Davies@kcl.ac.uk

URL: http://www.mth.kcl.ac.uk 